\title{
Effects of repeated administrations of atropine on two multiple schedules
}

RICHARD D. WILLIS ${ }^{2}$ and LARRY M. WINDLAND. ${ }^{3}$ University of Illinois, Urbana, Ill. 61801

Twenty $\mathrm{mg} / \mathrm{kg} I P$ injections of atropine were administered to albino rats after their responding had stabilized on mult $F R$ $80 \mathrm{DRL} 15 \mathrm{sec}$ or on mult VI $30 \mathrm{sec}$ FI $30 \mathrm{sec}$. The first injections of atropine depressed responding on the $F R, V I$, and $F I$ components and increased responding on the $D R L$ component. Replication injections consistently reproduced the effects on the FR and VI components but had inconsistent effects on the DRL and FI components.

Boren \& Navarro (1959) were the first to examine the effects of atropine sulphate on operant behavior which had stabilized under the control of different schedules of reinforcement. Using doses ranging from 0.1 to $4.0 \mathrm{mg} / \mathrm{kg}$, they reported reductions in response rates on fixed ratio performance and inconsistent effects on fixed-interval performance. Carlton (1963) found that doses of 2 to $6 \mathrm{mg} / \mathrm{kg}$ of atropine increased responding controlled by a DRL schedule. In both experiments the typical response patterns produced by the baseline schedules were disrupted.

The present experiment was conducted to replicate and to extend the data from those experiments by using an additional baseline schedule, variable-interval, and by employing a higher dose of atropine $(20 \mathrm{mg} / \mathrm{kg})$. Furthermore, the effects of repeated administrations were examined to assess the reliability of the drug effects.

\section{METHOD}

The Ss were four male naive Holtzman albino rats which weighed 300 to $360 \mathrm{~g}$ and were 90 days old at the beginning of the experiment.

The apparatus consisted of two modified Skinner boxes equipped with two retractable levers and associated programming and recording equipment.

The rats were gradually shaped according to standard procedures to the terminal performance levels. The terminal schedules were mult FR 80 DRL $15 \mathrm{sec}$ and mult VI $30 \mathrm{sec}$ FI $30 \mathrm{sec}$, with the components in both instances being switched every $15 \mathrm{~min}$. During each 15 -min period only the lever appropriate to a given component was inserted into the
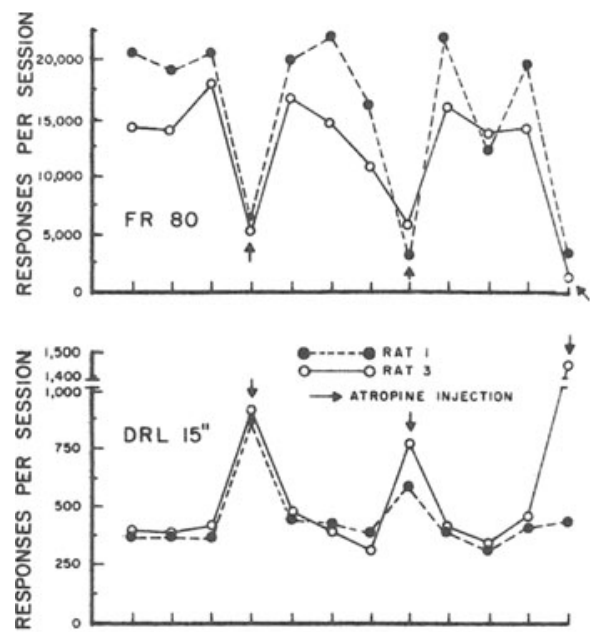

CONSECUTIVF SESSIONS

Fig. 1. Total responses per session for two rats on mult FR 80 DRL 15 sec.

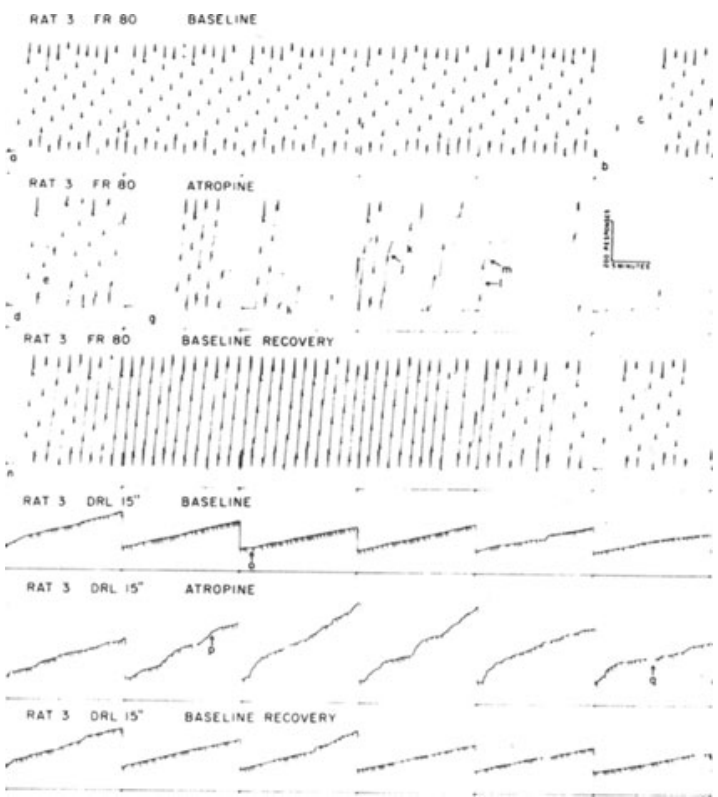

Fig. 2. Cumulative records of responding by one rat on mult FR 80 DRL $15 \mathrm{sec}$ for the day preceding (baseline), of (atropine) and following (baseline recovery) the first injection of atropine. See text for details.

Skinner box. Reinforcement was $0.01 \mathrm{cc}$ tap water. For a full description of such multiple schedule baselines, see Ferster \& Skinner (1957). The rats were exposed to the terminal contingencies for approximately $70 \mathrm{~h}$ prior to the first atropine injection. Daily sessions were $3 \mathrm{~h}$ long.

For Rats 1 and 3, on the mult FR 80 DRL $15 \mathrm{sec}$ schedule three days intervened between Injections 1 and 2 and between Injections 2 and 3. For Rat 4, on the mult VI $30 \mathrm{sec}$ FI $30 \mathrm{sec}$ schedule, eight days intervened between Injections 1 and 2 and four days between Injections 2 and 3. For Rat 5, on the same schedule, 10 days intervened between Injections 1 and 2 and four days between Injections 2 and 3. Rats were always given $2 \mathrm{~min}$ access to tap water following daily sessions.

Commercially available atropine sulphate was prepared for injection with sterile water to form a $40 \mathrm{cc} / \mathrm{kg}$ solution. All injections were $20 \mathrm{mg} / \mathrm{kg}$ administered intraperitoneally immediately before the beginning of a session. \section{RESULTS}

The results are presented in Fig. 1, 2, and 3. Figure 1 shows the number of responses per session on both components of the mult FR 80 DRL $15 \mathrm{sec}$ schedule. All injections of atropine reduced the overall response rates for both rats on the FR 80 component. Furthermore, baseline response levels were easily recovered.

Figure 2 shows cumulative records of responding for Rat 3 on the day immediately preceding ("baseline"), of ("atropine"), and immediately following ("baseline recovery") the first injection. The FR 80 baseline record is typical of the performance of both rats: A short pause at the beginning of the session (a); a pause at the beginning of the last exposure to the FR 80 component (b); and longer pauses after reinforcement near the end of the session (c). Otherwise baseline performance was characterized by high, steady response rates with little or no pausing after reinforcement.

The disruptive effects of atropine had an early onset and 


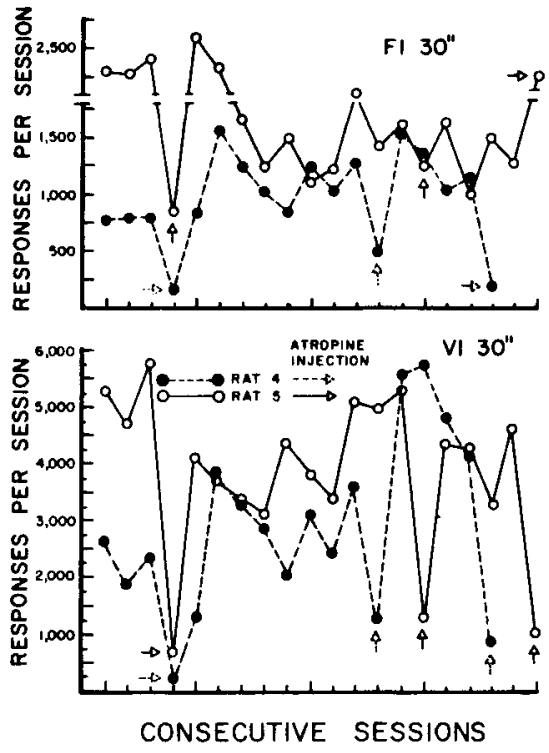

Fig. 3. Total responses per session for two rats on mult VI 30 sec FI 30 sec.

persisted throughout the session. The pause at the beginning of the session (d) was longer than usual. A ratio run with "rough" grain occurred at $e$ and an interrupted run at f. A long pause occurred at the beginning of the second exposure to the FR 80 component $(g)$. These effects $(e, f$, and $g$ ) never occurred in baseline. Another "rough" grain run occurred at $\mathrm{j}$; interrupted runs occurred at $h, k$, and $m$; long pauses such as at $i$ occurred frequently. Occasionally "normal" ratio runs did occur under atropine (1). The interrupted run (n) at the beginning of the session on the day following the injection was not typical of baseline performance. However, the rest of the session was typical.

Figure 1 also shows that the first atropine injection greatly increased response rates on the DRL $15 \mathrm{sec}$ component for both rats and that the baselines were easily recovered. Subsequent injections resulted in basically the same effect for Rat 3, but had decreasing effects for Rat 1 .

The DRL $15 \mathrm{sec}$ baseline and baseline recovery records in Fig. 2 are both representative of typical baseline performance. Long pauses such as at $q$ under atropine also occurred in baseline (o). Sustained responding at a moderate rate such as at $p$ under atropine never occurred in baseline.

Inspection of Fig. 2 shows clearly that, although atropine disrupted the response patterns which had stabilized on the two component schedules, in no instance did "ratio-like" responding occur under atropine in the DRL $15 \mathrm{sec}$ component, and in no instance did "DRL-like" responding occur in the FR 80 component. Thus, the disruptive effects of atropine occurred within but not between the individual component schedules.

Performance on the mult VI $30 \mathrm{sec}$ FI $30 \mathrm{sec}$ schedule is presented in Fig. 3. The first atropine injection reduced overall response rates for both rats on the FI $30 \mathrm{sec}$ component. For Rat 4, subsequent administrations had substantially the same effect on overall response rates; for Rat 5, however, subsequent administrations did not reduce the overall response rate. Since the effects were unreliable and the baseline difficult to recover, cumulative records are not presented. For the most part, the low overall rates under atropine were due to extended pauses. Occasionally there were scallops; occasionally there was responding early in the interval, followed by a pause.

The effects of atropine on the VI $30 \mathrm{sec}$ component were more consistent than on the FI $30 \mathrm{sec}$ component, and always resulted in a lowered overall response rate which was primarily due to long pauses. When responding did occur, it was in general at a low rate and had "rough" grain. As with the FI 30 $\mathrm{sec}$ component, baseline was difficult to recover, and cumulative records are therefore not presented.

\section{DISCUSSION}

An interesting finding in the present study was that the most consistent drug effects-both within and between Ss-were obtained for the component schedules which generated the highest response rates in baseline: FR 80 and VI $30 \mathrm{sec}$. For both components each injection clearly reduced the response rates to levels below the range observed in baseline. For responding on the other two schedules, DRL 15 sec and FI $30 \mathrm{sec}$, repeated injections had less clear effects: in both cases one animal behaved basically the same with repeated administrations, the other did not.

The differences observed in the present experiment were not related in any simple way to the ease with which baseline was recovered. For example, the DRL $15 \mathrm{sec}$ baseline was easy to recover and the VI $30 \mathrm{sec}$ baseline difficult to recover, yet inconsistent effects with repeated doses occurred with the former but not with the latter. The reverse relationship was obtained for the FR 80 (easily recovered) and FI $30 \mathrm{sec}$ (difficult to recover) components: repeated doses had consistent effects for the former but not for the latter.

Furthermore, these different effects cannot be ascribed to the time between injections since the multiple schedule baseline effectively controlled for that variable. For example, atropine always reduced the response rate on the FR 80 component for Rat 1 but had inconsistent effects on the DRL $15 \mathrm{sec}$ performance of the same rat.

Frequency of reinforcement can also be eliminated as a factor responsible for the observed effects. Frequency of reinforcement was the same for the VI $30 \mathrm{sec}$ and FI $30 \mathrm{sec}$ components by design, and it was fortuitously the same in the case of the FR 80 and DRL $15 \mathrm{sec}$ components: on FR 80 the number of daily reinforcers in baseline ranged from 146 to 272; on DRL $15 \mathrm{sec}$ from 165 to 203 for Rat 1.

Thus the must consistent relationship in these data was that the drug effects were more reliable on high-rate responding than on low-rate responding.

Although repeated administrations of atropine did not always have effects similar to the initial effects, the data from this experiment in general successfully replicate prior investigations (Boren \& Navarro, 1959; Carlton, 1963). Similarly, these data are for the most part consistent with the analysis proposed by Carlton (1963) to account for the most obvious effects of atropine on behavior: an increase in low-rate behavior and a decrease in high-rate behavior. The present experiment suggests that, in addition, the reliability of the effects may be a function of baseline response rate.

Subsequent research in this area might explore more parametrically the roles of dose level and specific parameters of the schedules in determining the effects of initial and subsequent injections of atropine. Furthermore, a variety of baseline reinforcers should be investigated since these effects may depend on motivational influences.

\section{REFERENCES}

BOREN, J. J., \& NAVARRO, A. P. The action of atropine, benactyazine and scopolamine upon fixed-interval and fixed-ratio behavior. Journal of the Experimental Analysis of Behavior, 1959, 2, 107-115.

CARLTON, P. L. Cholinergic mechanisms in the control of behavior by the brain. Psychological Review, 1963, 70, 19-39.

FERSTER, C. B., \& SKINNER, B. F. Schedules of reinforcement. New York: Appleton-Century-Crofts, 1957.

$$
\text { NOTES }
$$

1. This research was supported in part by USPH Grant NIMH 4853 to Dr. L. I. O'Kelly.

2. Reprints may be obtained from R. D. Willis, Comparative Psychology Laboratory, 129 N. Race, Urbana, Illinois 61801.

3. Present address: Southern Illinois University, Carbondale, Illinois 62901 . 